\title{
Acoustic Time-of-Flight Imaging of Polymer Electrolyte Membrane Water Electrolysers to Probe Internal Structure and Flow Characteristics
}

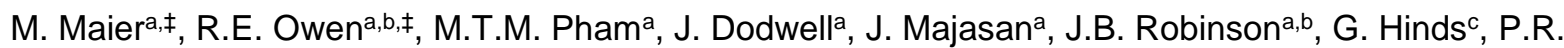 \\ Shearinga,b, D.J.L. Brett ${ }^{a, b^{*}}$ \\ a Electrochemical Innovation Lab, Department of Chemical Engineering, UCL, London, WC1E 7JE, \\ United Kingdom \\ ${ }^{b}$ The Faraday Institution, Quad One, Harwell Science and Innovation Campus, Didcot, OX11 ORA, UK \\ c National Physical Laboratory, Hampton Road, Teddington, Middlesex, TW11 OLW, UK \\ ¥ These authors contributed equally to this work. \\ "Corresponding author. Tel.: +44 (0)20 7679 3310; fax: +44 (0)20 7383 2348. E-mail address: \\ d.brett@ucl.ac.uk (D.J.L.Brett).
}

\section{Abstract}

Acoustic time-of-flight (ATOF) imaging has been demonstrated as a low-cost, rapid, non-destructive, operando tool to characterize processes in the flow channels and liquid-gas diffusion layer (LGDL) of polymer electrolyte membrane water electrolysers (PEMWEs). An array of 64 piezoelectric sensors was used, with all sensors emitting input pulses and detecting the acoustic wave reflected by the sample (pulse-echo mode). The shape and intensity of this reflected waveform depends on the ratio of reflection and transmission at phase interfaces and is strongly affected by resonant scattering of acoustic waves by gas bubbles. This AToF imaging technique was deployed to produce reflection intensity maps of the anode flow-field and LGDL; by measuring the AToF response for current densities ranging from $0.00 \mathrm{~A} \mathrm{~cm}^{-2}$ to $2.00 \mathrm{~A} \mathrm{~cm}^{-2}$, a close correlation was found between the acoustic attenuation in the flow-field and the production and removal of oxygen gas through the flow channels. Furthermore, a close link between the AToF response and water thickness in the LGDL was demonstrated, as supported by literature data. The application of the AToF technique has been established as a novel way of investigating PEMWE operation and as an alternative to more complex imaging techniques such as neutron imaging.

Keywords: Acoustic Time-of-Flight; PEM Water Electrolyser; Mass Transport; 3D Imaging; Diagnostics 


\section{Introduction}

Electrochemical energy conversion and storage devices are an integral part of modern life and hold great promise for the transformation of our energy system. While battery technology has been the focus of public awareness in recent years, hydrogen-based conversion and storage is increasingly viewed as a viable alternative for many applications [1]. The commercially dominant hydrogen production technology is steam methane reforming, but carbon-free, 'green' hydrogen synthesis is predominantly achieved by water electrolysis [2]. Polymer electrolyte membrane water electrolysers (PEMWEs) offer a number of advantages over the commercially more mature alkaline electrolysis. These include higher attainable current density, improved safety due to reduced gas crossover, and a more compact design. Further, the increasing research and commercial uptake of PEM fuel cells create synergistic effects driving the development of PEMWEs [2].

As PEMWEs mature, capital investment costs are expected to drop further $[3,4]$ and novel materials and engineering approaches reduce operational voltage and therefore allow for much higher current densities $[5,6]$. These developments will accelerate the industrial and commercial uptake of PEMWEs $[1,7]$.

The widespread industrial use of PEMWEs for hydrogen production and grid stabilization will be accelerated through advances in the performance and cost of the technology. One of the most effective ways of improving such technology is through the establishment and use of a robust set of metrology tools to screen the effect of design modification, support optimization of operational parameters and facilitate low-cost maintenance and repair. Traditional electrochemical testing methods, including the interpretation of polarization curves and electrochemical impedance spectroscopy, are valuable diagnostic techniques, but do not offer the same level of spatially resolved information as modern imaging technologies. X-ray computed tomography [8-11], neutron imaging [12-16] or optical imaging [17-20] have been used to visualise internal operational processes such as the two-phase flow in the flow channels, the microstructure of the liquid-gas diffusion layer (LGDL), water-gas dynamics in the LGDL, or the particle structure of the catalyst layer. However, these 
techniques are often expensive and may require adaptations of the PEMWE cell to facilitate measurement.

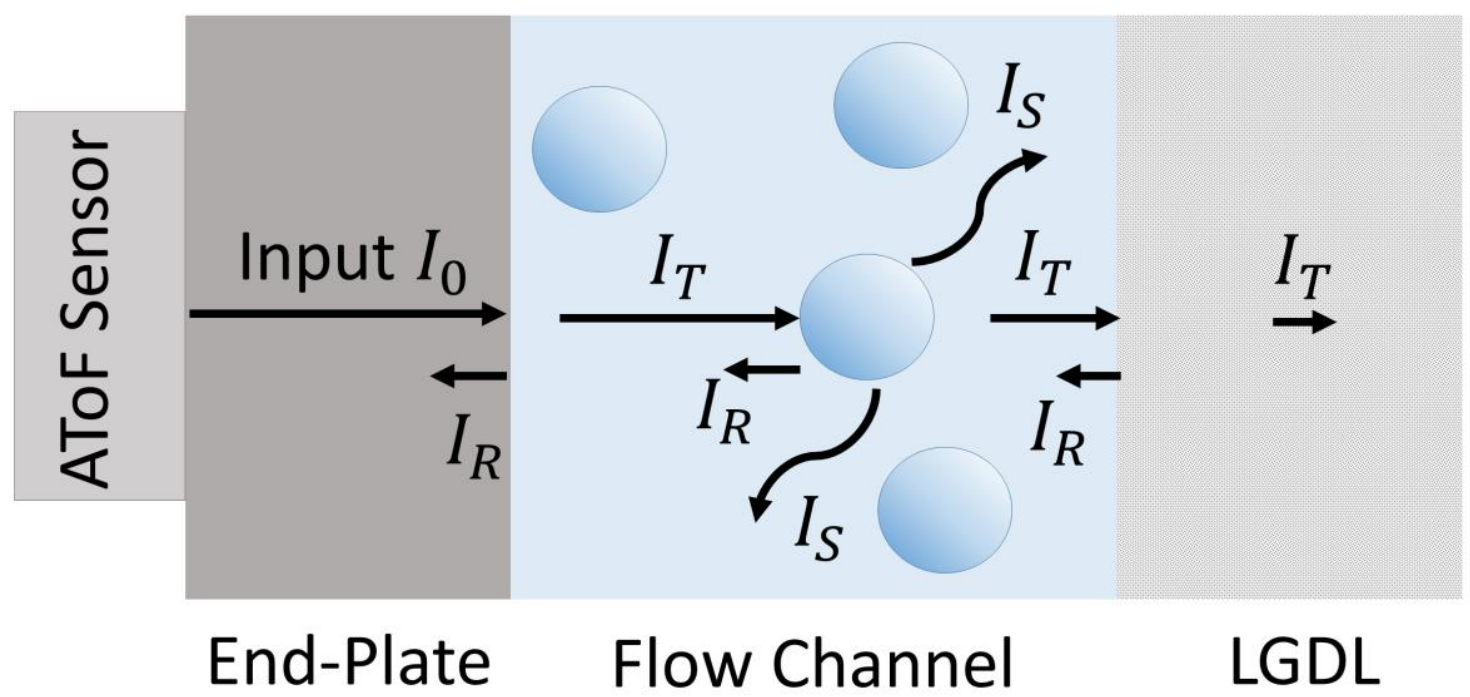

Fig. 1: Schematic diagram of acoustic attenuation mechanisms in a PEMWE. The input signal $\left(I_{0}\right)$ is emitted by the AToF sensor and penetrates the end-plate. At each phase interface, a portion of the signal is reflected $\left(I_{R}\right)$, while the remaining signal is transmitted through the next domain $\left(I_{T}\right)$. In the flow channels, the signal can be reflected at liquid/gas interfaces $\left(I_{R}\right)$, but also attenuated by resonant scattering with gas bubbles $\left(I_{S}\right)$. Arrow lengths indicate the relative intensity of an acoustic signal.

Acoustic diagnostic techniques can overcome many of these disadvantages and promise low-cost, fast, non-destructive operando analysis of PEMWEs and other electrochemical energy storage and conversion devices. Acoustic diagnostics can be differentiated into passive acoustic emission (AE) and active acoustic time-of-flight (ATOF) techniques. AE relies on detecting mechanical perturbations emitted by objects using a piezoelectric sensor and is routinely applied in monitoring of mechanical stability and structural health in the construction sector $[21,22]$. It has also found applications for fuel cells and batteries, which range from monitoring particle cracking and lithium intercalation in Li-ion batteries [23-25] and characterizing seal stability for solid oxide fuel cells $[26,27]$ to observing various processes in PEM fuel cells [28-30]. Maier et al. [31,32] applied AE to PEMWEs and demonstrated its 
ability to monitor the processes in the flow channels, including qualitatively detecting bubble number and size over current density, in real-time.

In contrast to $\mathrm{AE}, \mathrm{AToF}$ is an acoustic technique wherein samples are actively probed with ultrasound waves; the transmitted or reflected response signal is recorded as a function of time-of-flight (ToF) through the sample. The intensity of a reflection signal depends on how strongly the input signal has been attenuated by the sample. Acoustic attenuation occurs at an interface between two different materials due to reflection or resonant scattering, for example from a gas bubble or group of bubbles (Figure 1). At each interface a portion of the incoming signal is reflected, while the remaining signal is transmitted into the next domain. When an acoustic signal interacts with a bubble interface, it is not only partially reflected, but can also be resonantly scattered if the signal frequency is close to the resonant frequency of the bubble. In this case the signal is scattered in all directions, causing a very high acoustic attenuation of the input signal.

AToF was first introduced to electrochemical energy storage and conversion devices by Sood et al. [33], who successfully used AToF transmission measurements to detect interface degradation in a cycled Li-ion pouch cell. Hsieh et al. [34], who conducted transmission and reflection AToF experiments on cylindrical and pouch Li-ion and alkaline battery cells, demonstrated that changes in the time-of-flight of the acoustic signal are linked to physical changes in the electrodes (density and modulus), showed that these changes can be used to measure the state-of-charge of the battery, and validated their results against a computational model of sound propagation in a series of battery electrodes. This work was later advanced to show the ability of AToF to detect Zn gel dehydration and $\mathrm{ZnO}_{2}$ formation in $\mathrm{Zn} / \mathrm{MnO}_{2}$ batteries [35], to measure the effective stiffness of Li-ion batteries [36], as well as to develop a supervised machine learning technique to predict battery state-of-charge from the ultrasonic time-of-flight [37]. Measuring state-of-charge has been demonstrated by other authors as well, using AToF $[38,39]$ or guided acoustic waves $[40,41]$. Robinson et al. $[42,43]$ combined AToF mapping across the surface of a Li-ion pouch cell with X-ray computed tomography to further demonstrate its ability to elucidate internal battery structure and investigated anode and cathode 
structure in detail during charge and discharge. This demonstrated the use of AToF to observe mechanical processes and changes, such as diagnosing lithium plating in Li-ion batteries [44] and mechanical defects in electrode structures [45].

This work, for the first time to our knowledge, applies AToF for fast, non-destructive, and operando imaging of PEMWEs. Where previous work on AToF analysis of batteries has mostly relied on single sensors, an array of 64 sensors was deployed in this work. Reflection (pulse-echo) measurements were used to investigate gas/water (two-phase) processes occurring in the flow-field and LGDL as a function of current density. This provides insights into mass transport phenomena in PEMWEs, which would otherwise have required more expensive and time-consuming diagnostic approaches.

\section{Experimental}

\subsection{PEMWE Cell}

The PEMWE used for experiments (Figure $2(\mathrm{a})$ and (b)) had a square $9 \mathrm{~cm}^{2}$ active area and consisted of acrylic end-plates, titanium flow-fields, a titanium LGDL on the anode side, a carbon paper gas diffusion layer (GDL) on the cathode side, and a catalyst coated membrane (CCM). The flow-fields consisted of nine parallel channels with a length of $3.00 \mathrm{~cm}$, a width of $1.76 \mathrm{~mm}$, and a depth of 2.00 $\mathrm{mm}$. Sintered titanium powder (Merelex, USA) was used as LGDL and Toray H-060 carbon paper as GDL. The CCM was coated with $3 \mathrm{mg} \mathrm{cm}^{-2}$ iridium/ruthenium oxide on the anode side and $0.6 \mathrm{mg} \mathrm{cm}^{-2}$ platinum on carbon on the cathode side (ITM Power, UK). The cell was held together with eight M5 screws, each fastened to a torque of $2.5 \mathrm{Nm}$. For all experiments, water was circulated through anode and cathode with an inlet temperature of $50{ }^{\circ} \mathrm{C}$ and a flow rate of $50 \mathrm{ml} \mathrm{min}^{-1}$ at each electrode.

A Gamry Reference 3000 Galvanostat/Potentiostat with a Gamry 30k Booster (Gamry Instruments, USA) was used for electrochemical testing between $0.00 \mathrm{~A} \mathrm{~cm}^{-2}$ and $2.00 \mathrm{~A} \mathrm{~cm}^{-2}$. The $i-V$ curve was obtained following the guidelines of the Horizon 2020 Fuel Cells and Hydrogen Joint Undertaking Programme [46]. Electrochemical impedance spectroscopy (EIS) data were recorded in the frequency range from $10 \mathrm{mHz}$ to $10 \mathrm{kHz}$ at specific $\mathrm{DC}$ current densities between $0.00 \mathrm{~A} \mathrm{~cm}^{-2}$ and $2.00 \mathrm{~A} \mathrm{~cm}^{-2}$ with an AC current modulation of $10 \%$ of the applied DC current. 
(a)

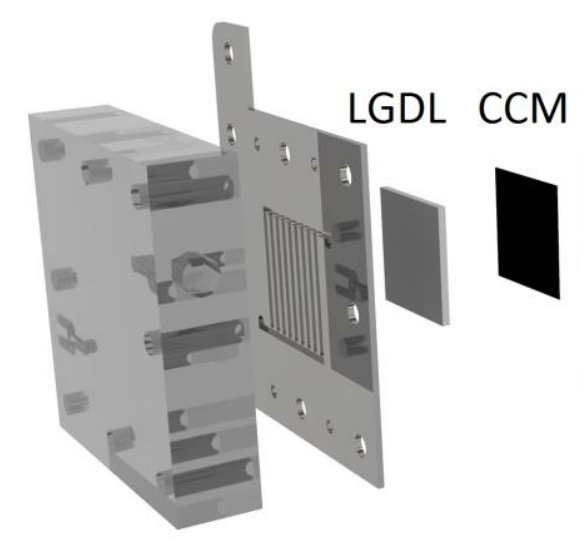

Flow-Field (b)
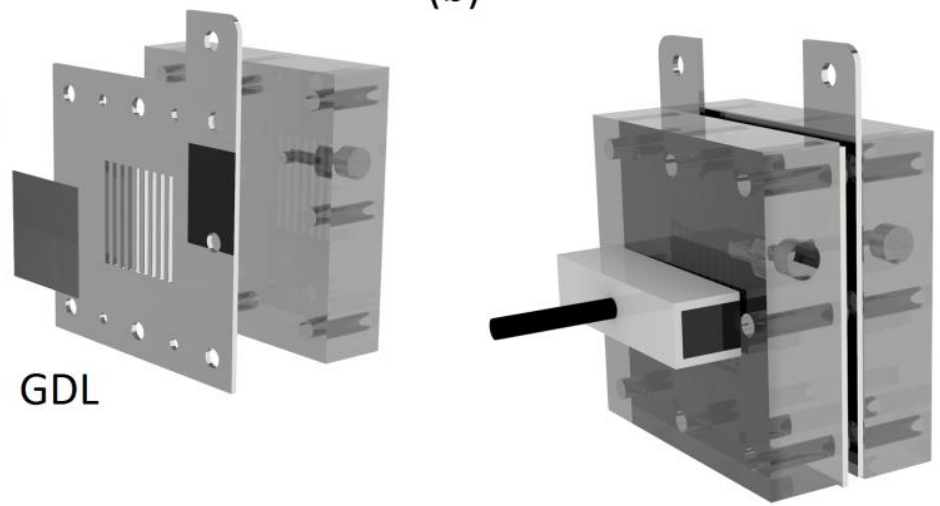

\section{End-Plate}

Fig. 2: (a) The PEMWE setup consists of end-plates, parallel flow-fields, a titanium sintered liquid-gas diffusion layer (LGDL), the catalyst coated membrane (CCM), and the gas diffusion layer (GDL) on the cathode side. (b) The AToF sensor array is mounted on the outer surface of the anode end-plate.

\section{$2.2 \quad$ AToF Measurements}

All measurements were taken with a near-wall phased array (length: $66 \mathrm{~mm}$, width: $19 \mathrm{~mm}$, height: $25 \mathrm{~mm}$ ) of 64 acoustic sensors (Model 10L64-NW1, Olympus Scientific Solutions, USA) in pulse-echo mode on the anode side of the PEMWE. Each data point was averaged from the signals received from 16 neighbouring sensors $(1-16,2-17,3-18, \ldots . .49-64)$, which is called a 'sensor unit'. Each sensor unit recorded one measurement per second, and the signal of two neighbouring sensor units was averaged so that 33 spatially resolved data points were obtained for each measurement. The signal received was the reflected portion of the input signal, which was a pulse sequence with a maximum repetition frequency of $20 \mathrm{kHz}$. All data was amplified before recording. The amplitude of this amplification is called the acoustic gain. Sensors were controlled and read out with an Olympus FOCUS PX system (Olympus Scientific Solutions, USA). All AToF data in this work is given as a percentage of the sensor saturation value. Before each experiment, a layer of acoustic gel (D12 Couplant Gel, Olympus Scientific Solutions, USA) was applied to the outer surface of the PEMWE and the sensor. This provided good acoustic coupling between sensor and PEMWE surface and ensured a consistent signal across the surface. 
Two different types of AToF experiments were conducted: fixed position experiments and area scans. For fixed position experiments, the sensor was mounted to a specific location and data were recorded continuously while the current density was increased from $0.00 \mathrm{~A} \mathrm{~cm}^{-2}$ to $2.00 \mathrm{~A} \mathrm{~cm}^{-2}$ in $0.10 \mathrm{~A} \mathrm{~cm}^{-2}$ increments, each lasting $120 \mathrm{~s}$. Three different locations along the length of the flow-field were defined, each covering the whole width of the flow-field. The lower location was $0.5 \mathrm{~cm}$ from the bottom end of the active area, the upper location $0.5 \mathrm{~cm}$ from its top end, and the middle location exactly $1.5 \mathrm{~cm}$ from either end of the flow-field. On the other hand, area scans were performed by moving the sensor array across the active area $\left(10 \mathrm{~mm} \mathrm{~s}^{-1}\right)$ from the bottom to the top of the flow channels, while operating the PEMWE at a constant current density. The sensor array was mounted onto an additional Rexolite block (thickness: $2 \mathrm{~cm}$ ) to avoid damaging the sensor surface while gliding it over the PEMWE surface.

To analyse the acoustic response from different components of the PEMWE, different time-of-flight intervals of the echo-response were analysed; as the LGDL was located after the flow-field, peaks at a higher ToF were attributed to the LGDL, lower ToF peaks were attributed to the flow-field. Experiments were conducted with different acoustic gains (signal amplification). A clear signal from the anode flow-field was obtained at a gain of $40 \mathrm{~dB}$, however, this acoustic intensity was not strong enough to obtain a clear reflection signal from the LGDL. Therefore, experiments were also carried out at a gain of $60 \mathrm{~dB}$, in which case the signal from the flow-field was saturated, but sufficient signal intensity for analysis was obtained from the LGDL. Preliminary experiments demonstrated that, at a chosen acoustic gain, the relevant time-of-flight intervals were not saturated for the current densities between $0.00 \mathrm{~A} \mathrm{~cm}^{-2}$ and $2.00 \mathrm{~A} \mathrm{~cm}^{-2}$ applied in this work.

For comparison to AToF measurements of the PEMWE during operation, measurements of the dry PEMWE cell (without water inflow) at all three different sensor locations were taken as well. 


\subsection{AToF Data Analysis}

Data was visualised in real-time in the FocusPC software (Olympus Scientific Solutions, USA), which was also used for data export. The FocusPC interface was used to measure the maximum reflection intensity in time-of-flight intervals containing the reflection peaks related to specific components of the PEMWE, e.g. flow-fields or the LGDL. The waveform intervals correlated to components of the PEMWE were chosen manually based on a number of AToF scans with varying parameters. Subsequently, the peak intensity was recorded for each sensor unit and throughout the duration of the experiment.

The resulting data points were mapped and analysed qualitatively and quantitatively. Each peak of the waveform (intensity value in an acoustic map) is caused by the reflection of the acoustic input signal at an interface. The intensity of a reflection peak relates to the fraction of signal which is reflected at a given interface (the acoustic reflection coefficient $R$ ), while the fraction of acoustic signal which is not reflected is the transmission coefficient $T$. The acoustic reflection (Equation (1)) and transmission (Equation (2)) coefficients are a function of the acoustic impedances $z$ of the phases 1 and 2, which form the interface [47].

$$
\begin{aligned}
& R=\frac{z_{1}-z_{2}}{z_{1}+z_{2}} \\
& T=\frac{2 z_{1}}{z_{1}+z_{2}}
\end{aligned}
$$

The Equations (1) and (2) illustrate that the signal is not reflected if both phases at the interface exhibit the same acoustic impedance. Increasing difference in acoustic impedance results in a consequent increase in the intensity of reflection. The acoustic impedance of a material is a function of material and acoustic wave properties as well as a wide range of physical conditions [47]. For many materials, $Z$ is the product of density and the speed of sound in that material $[42,47]$, illustrating that materials are likely acoustically matched when they exhibit similar physical properties. This implies that signal attenuation is more pronounced at a solid/gas interface than at a liquid/gas interface. 
Understanding the causes of reflection in a system enables an understanding of the nature of an interface from observing the intensity of the recorded reflection peaks. However, the pulse-echo signal is not only influenced by reflection at interfaces, but also by resonant scattering. This occurs when an acoustic wave travels through a medium containing gas bubbles (such as water in the flow channels of a PEMWE). If the frequency of the wave is at or near the resonance frequency of a gas bubble, a major fraction of the wave is scattered. According to Equation (3), the resonance frequency $f_{r}$ of a bubble is, among others, a function of its radius $r[48,49]$.

$$
f_{r}=\frac{1}{2 \pi} \sqrt{\frac{3 \gamma p_{\infty}}{\rho_{l} r^{2}}}
$$

where the ambient pressure is $p_{\infty}, \gamma$ is the polytropic coefficient, and the density of the liquid is $\rho_{l}$.

During operation of a PEMWE, a multitude of gas bubbles are produced, covering a wide range of bubble sizes; hence, a wide range of frequencies exist within which resonant scattering between acoustic wave and bubbles can occur. However, this multitude of bubbles can be described as a group of coupled oscillators, exhibiting collective oscillation modes, and is commonly referred to as bubble cloud [50]. The resonant frequency of a bubble cloud is not the summation of all individual bubble resonance frequencies, but a specific resonant frequency due to the collective oscillation $[48,50,51]$, which, for a spherical cloud of individual bubbles (radius $r_{c}$ ), can be calculated depending on the fraction of gas, $\chi$, in the cloud [48].

$$
f_{r}=\frac{1}{2 \pi} \sqrt{\frac{3 \gamma p_{\infty}}{\chi \rho_{l} r_{c}^{2}}}
$$

In Equation (4), the polytropic coefficient is usually taken as 1.0 for isothermal operation and 1.4 for adiabatic operation [48]. The operation of a PEMWE in a realistic scenario will fall between these two extreme cases. 
In the following, Equation (4) will be used to calculate the resonant scattering frequency of bubbles in the flow channels of a PEMWE. During operation, the flow channels are typically filled with a multitude of gas bubbles, so that the use of a single bubble model (Equation (3)) for the characterisation of PEMWE flow channel scattering frequency appears inadequate. The use of the bubble cloud model for the calculation of the resonant scattering frequency is well established $[48,51,52]$.

The radius of a bubble cloud will, in the following, be assumed to be identical to the width of a flow channel, as previous work has shown that at any current density the generated gas bubbles are scattered across the width of the entire channel [31]. The ambient pressure will be assumed to be atmospheric, as the PEMWE is operated with minimal excess pressure, and the density of the liquid is taken as the density of water. The calculation of the gas fraction in the flow channels is described elsewhere [31,53].

To obtain frequency information from the pulse-echo response, fast Fourier transform (FFT) analysis of the AToF waveform was performed using the FocusPC software. The pulse-echo response across the entire time-of-flight was analysed, yielding an intensity spectrum as a function of frequency. The frequency at which the maximum intensity was found, will be referred to as peak frequency in the following. Comparison of the peak frequency with values obtained from Equation (4) serves to indicate whether the obtained signal predominantly originated from resonant scattering.

\section{Results and Discussion}

\subsection{Electrochemical Testing}

The PEMWE cell showed typical polarization behaviour (Figure 3 (a)), consistent with previously reported data sets $[17,54-56]$. EIS was used to examine the influence of mass transport effects on the overall PEMWE performance (Figure 3 (b)): while the width of the left-hand arc (high-frequency) in the Nyquist plot determines the activation overpotential, the right-hand arc (low-frequency) indicates the magnitude of the mass transport overpotential $[54,57,58]$, even though inductive effects, as observed for $0.25 \mathrm{~A} \mathrm{~cm}^{-2}$ and $1.00 \mathrm{~A} \mathrm{~cm}^{-2}$, can make an exact quantification difficult. The width of the 
right-hand arc increased from low to high current density, by around $30 \%$ from $0.25 \mathrm{~A} \mathrm{~cm}^{-2}$ to $2.00 \mathrm{~A}$ $\mathrm{cm}^{-2}$. This indicates a significantly higher overpotential due to mass transport processes at higher current densities.
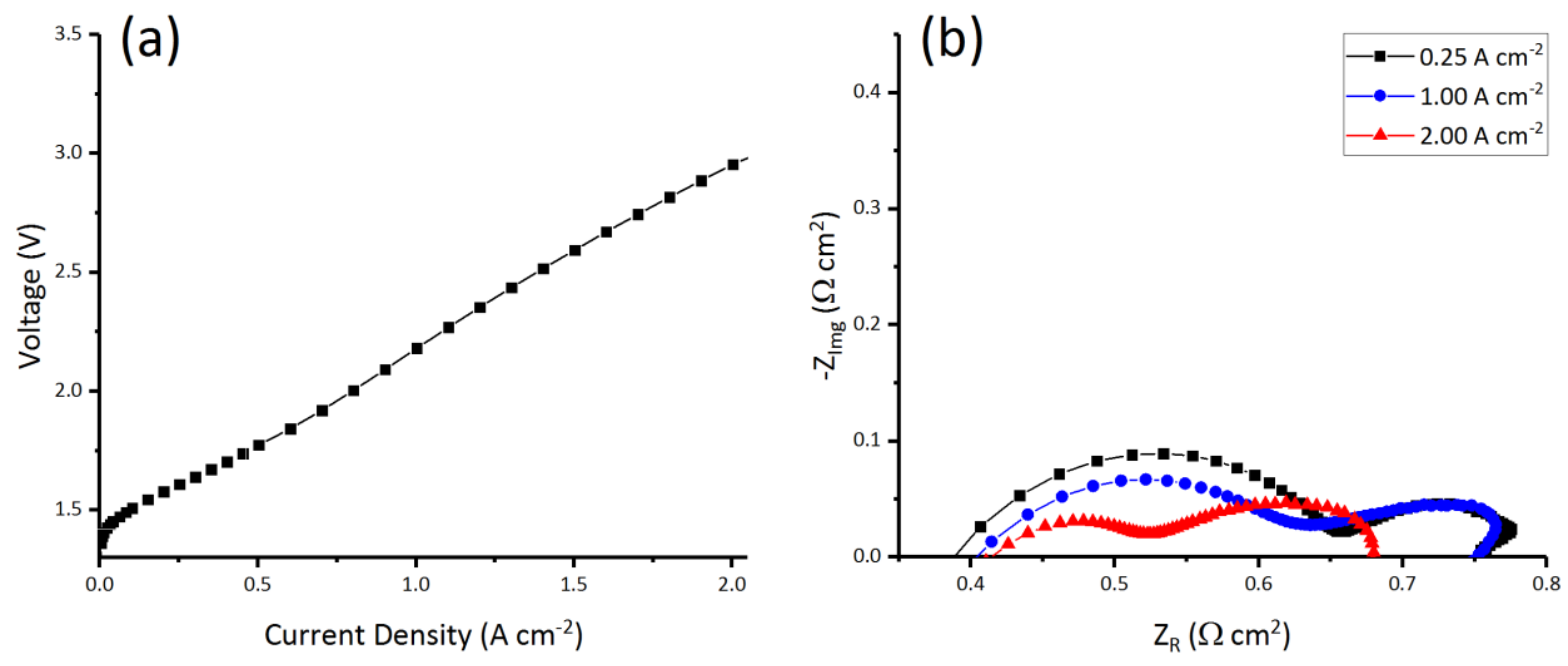

Fig. 3: (a) Overall PEMWE cell performance, shown as voltage against current density ranging from $0.00 \mathrm{~A} \mathrm{~cm}^{-2}$ to $2.00 \mathrm{~A}$ $\mathrm{cm}^{-2}$. (b) Electrochemical impedance spectra of the PEMWE between $0.1 \mathrm{~Hz}$ and $10 \mathrm{kHz}$, represented as negative imaginary impedance $-Z_{\text {img }}$ against the real impedance $Z_{r}$ (Nyquist plot), for $0.25 \mathrm{~A} \mathrm{~cm}^{-2}, 1.00 \mathrm{~A} \mathrm{~cm}^{-2}$, and $2.00 \mathrm{~A} \mathrm{~cm}^{-2}$.

On the anode side of a PEMWE, as current density increases, an increasing flux of water has to be transported to the active catalyst sites, while increasing amounts of oxygen have to be removed from the system. The water transport capacity from the flow-field, through the porous LGDL, to the CCM and oxygen transport in the reverse direction is finite and consequently increasing mass transport inefficiencies occur with increasing current density and lead to an increase in PEMWE operational voltage. This rise is due to the mass transport overpotential, which is caused by the overall increase of mass transport in the PEMWE system.

\subsection{Structural Investigation with AToF}

The ability of acoustic waves to penetrate through layers of material can be used for rapid inspection and diagnostics. AToF scanning of the entire active area (as described in Section 2.2) provided a spatially resolved 3D imaging technique, visualising the structure of different PEMWE components. Experiments at two different acoustic gains were used to investigate the structure and assembly of 
flow-field and LGDL in the PEMWE. Each AToF experiment offered depth-profiling of the same layers of the PEMWE, such as flow-field and LGDL, but investigation of different layers was carried out in separate experiments to obtain an optimal and clear signal from each component.

(a)

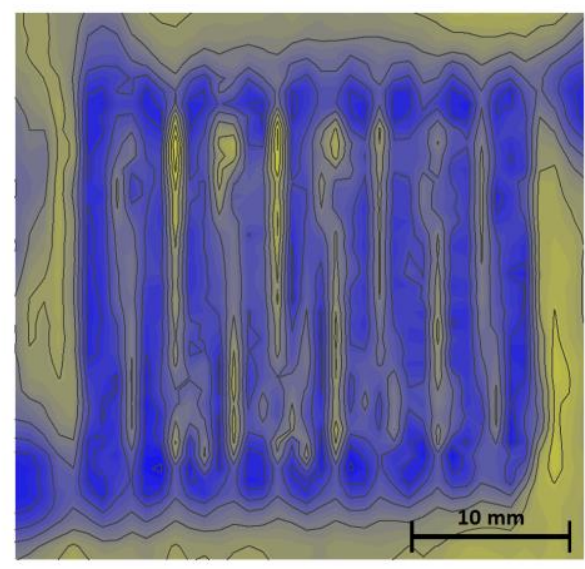

(c)

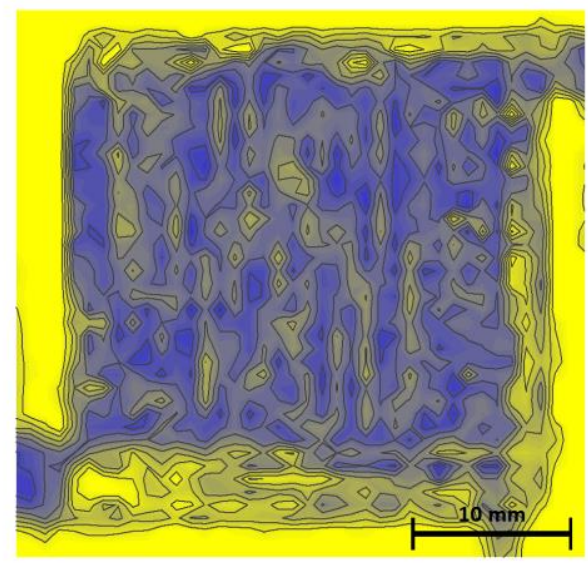

$\%$

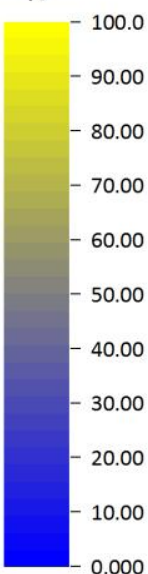

$\%$

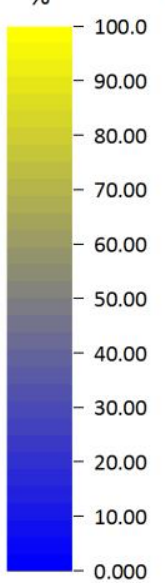

(b)

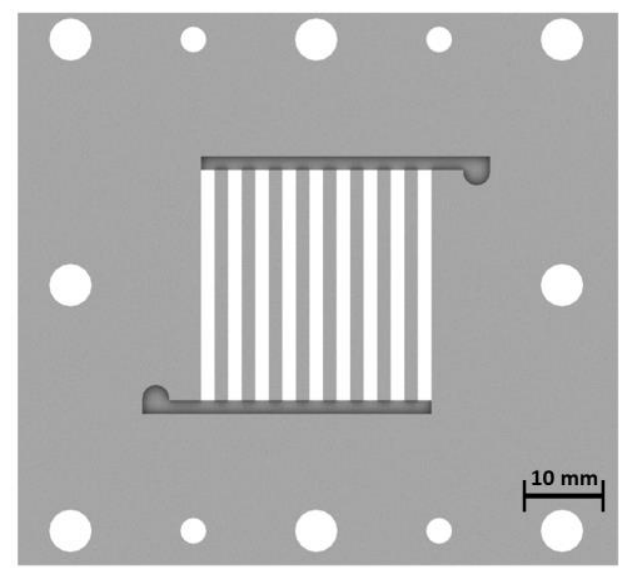

(d)

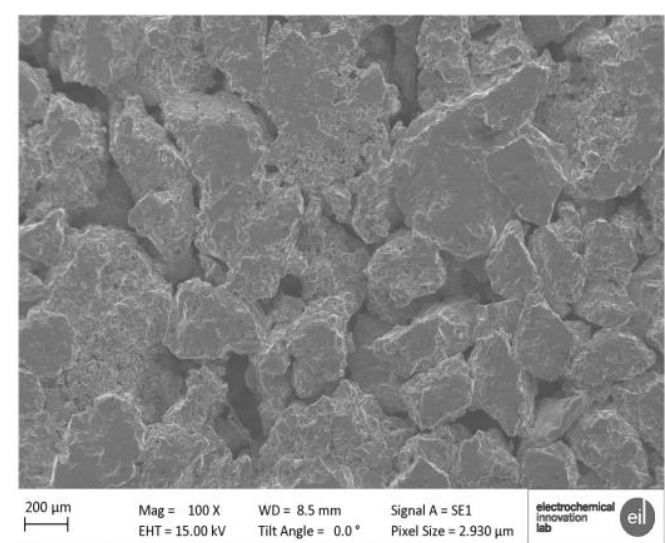

Fig. 4: (a) AToF scan of the anode flow-field of the PEMWE, showing rib and channel areas, as well as water inlet and outlet. (b) The CAD design of the flow-field, shown for comparison. (c) AToF scan of the LGDL, illustrating a granular distribution of high and low reflection intensity. (d) SEM image of the LGDL microstructure given for comparison. Image was obtained on a Zeiss EVO MA10 (Carl Zeiss, USA) at an electron accelerating voltage of $15 \mathrm{kV}$ and a magnification of 100x. Both AToF area scans were obtained during operation of the PEMWE at a constant current density of 0.25 A cm-2. Hence, water was circulated through the flow-field, and LGDL and flow channels contained water as well as oxygen.

The PEMWE was operated at $0.25 \mathrm{~A} \mathrm{~cm}^{-2}$ for both experiments, hence water and oxygen gas both exist in flow-field as well as the pore phase of the LGDL. Based on preliminary experiments, different timeof-flight intervals were chosen for each component, measuring the maximum reflection peak intensity 
in the respective interval. The resulting reflection intensity maps are shown in Figure 4 and should be understood as approximate representations of the investigated physical structure. A more detailed and quantitative investigation of the reflection characteristics of flow-field and LGDL can be found in Section 3.3.

The structure of the flow-field was visualized with AToF, showing details of the flow channels, the ribs and water inlet and outlet. A low reflective peak was observed in channel areas (blue areas in Figure $4(a))$, while the ribs were marked by high reflective peaks (yellow areas). Comparison between the actual geometry of the parallel flow-field (Figure 4 (b)) with the AToF image showed that all eight ribs and nine channels were detected. The ratio between rib length and width was 17.05 for the actual flow-field design, and was manually measured to be 18.70 from the AToF image. Based on the width of the active area ( $30 \mathrm{~mm}, 32$ pixel) a spatial resolution of $0.94 \mathrm{~mm}$ was obtained. The fact that high reflection intensities were detected in the rib areas, while lower intensities were found in the channel areas, suggests that the acoustic impedance of the acrylic end-plate was more closely matched with the acoustic impedance of the water-filled channels than with the acoustic impedance of the titanium flow-field ribs. This might seem counter-intuitive, but can be derived from the material values (Section 2.3). Further, the very low reflection intensity in the flow channels is likely to be attributed to resonant scattering from oxygen bubbles. This will be further validated in Section 3.3.

Investigating a different set of peaks at a higher acoustic time-of-flight (hence relating to a PEMWE component located behind the flow-field), an AToF image of the LGDL was obtained during operation of the PEMWE at $0.25 \mathrm{~A} \mathrm{~cm}^{-2}$ (Figure 4 (c)). The LGDL was a porous sintered titanium structure (Figure 4 (d)) with pores being filled with either water or gas. For the AToF response, a granular distribution of high and low reflective peaks, randomly distributed over the LGDL area, was observed. AToF is a depth-profiling technique, hence the reflection intensity from the LGDL was affected by acoustic processes in the flow-field and end-plate. As the reflection at an interface is a function of the acoustic impedance of both materials at this interface, the acoustic response from LGDL pores and particles 
was different in rib and land areas. This makes it difficult to clearly assign low or high reflection peaks to either pores or particles. Further, the granularity of the AToF response was much coarser than the microstructure of the LGDL. As observed above, the spatial resolution of the AToF measurement is $0.94 \mathrm{~mm}$, while the size of pores and particles of the LGDL are significantly smaller (Figure 4 (d)). This suggests that with the given experimental equipment AToF cannot be used to distinguish individual pores or particles, but rather larger accumulations of gas or water in a network of adjacent pores. However, by observing the overall reflection intensity as a function of current density, conclusions can be drawn on the volume averaged macroscopic processes in the LGDL (Section 3.3).

The above results show that AToF scans are a powerful 3D imaging and depth-profiling tool for quick inspection and qualitative analysis, and further examples of imagining the structure of the flow-field and LGDL at $0.00 \mathrm{~A} \mathrm{~cm}^{-2}$ and $2.00 \mathrm{~A} \mathrm{~cm}^{-2}$ can be found in Figure $\mathrm{S} 1$ in the Supplementary Information. However, to obtain quantitative data as a function of current density, fixed position AToF measurements are also investigated below to reduce any error that may be introduced while moving the acoustic sensor.

\subsection{AToF for Quantitative Assessment}

By applying AToF across the whole width of the active area at a fixed location, while varying current density, statistically reliable data (probing of a constant state of operation for $120 \mathrm{~s}$ ) on the acoustic response was obtained. Experiments were conducted at three different sensor locations (lower, middle, and upper as defined in Section 2.2) and two different acoustic time-of-flight intervals to access the flow-field and LGDL, respectively (Section 2.2). 
(a)

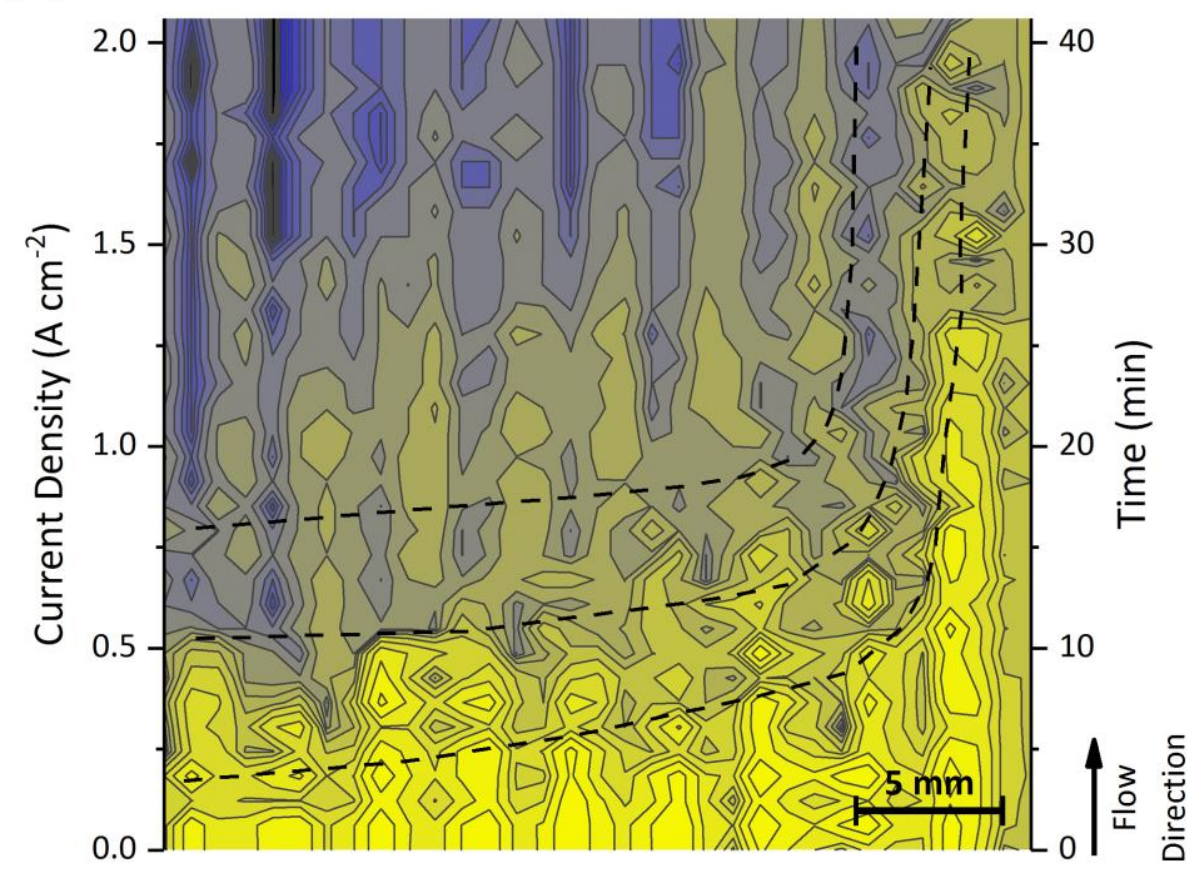

(b)

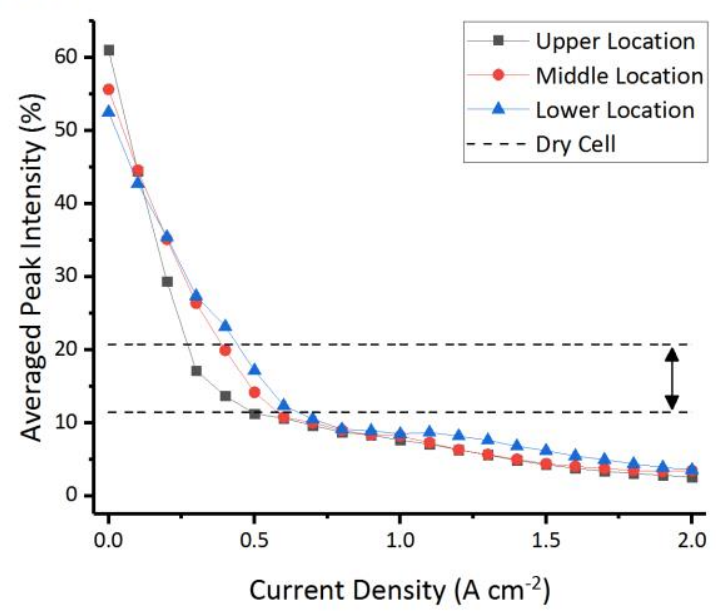

(c)

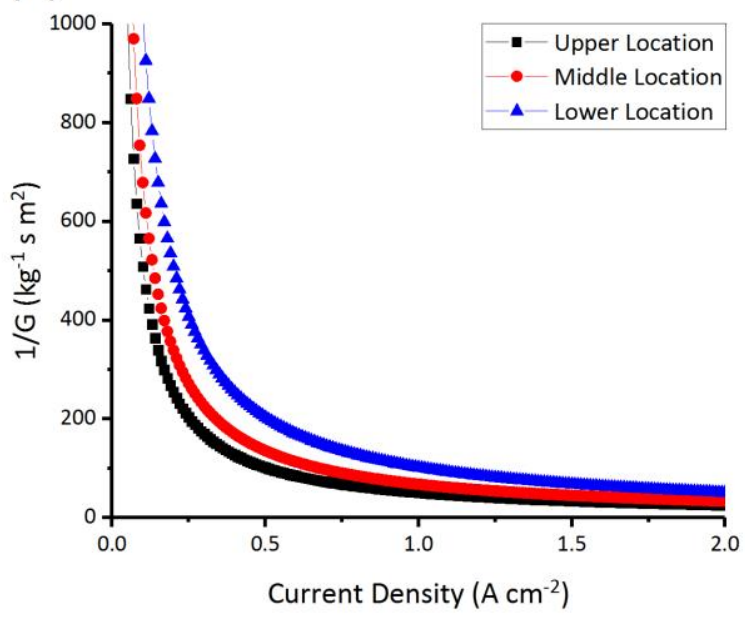

Fig. 5: (a) AToF reflection map of the flow-field across all flow channels at the middle sensor location for current densities ranging from $0.00 \mathrm{~A} \mathrm{~cm}^{-2}$ to $2.00 \mathrm{~A} \mathrm{~cm}^{-2}$. The reflection intensity was measured every second across the width of the active area, while the current density was increased in $0.10 \mathrm{~A} \mathrm{~cm}^{-2}$ intervals lasting $120 \mathrm{~s}$. This illustrates the evolution of the reflection intensity in a segment across the width of the flow-field, roughly the same height as the AToF sensor, as a function of current density. The intensity of the reflection peaks is indicated using a logarithmic color map. Dashed black lines indicate parabolic lines of identical intensity. (b) The average intensity of reflection peaks across all flow channels for lower, middle, and upper sensor locations as a function of current density. The average intensity band measured in the dry cell is shown for comparison (dashed black lines). (c) For comparison, the inverse of the amount of oxygen per flow channel, $G$, is shown as a function of current density for all three sensor locations. 


\section{Flow-Field}

Figure 5 (a) shows the AToF response from the flow-field at the middle location on a logarithmic scale between $0.00 \mathrm{~A} \mathrm{~cm}^{-2}$ and $2.00 \mathrm{~A} \mathrm{~cm}^{-2}$, hence, illustrating the evolution of the reflection intensity in the middle segment of the flow-field across the entire active area (and all flow channels and ribs) as a function of current density. To visualize this large relative changes in intensity across the current density range, the use of a logarithmic scale was required. A general trend from high reflection intensity to low reflection intensity was observed as current density was increased. While the average intensity of the flow-field reflection peaks was around $55 \%$ at $0.00 \mathrm{~A} \mathrm{~cm}^{-2}$, it dropped to $5 \%$ at $2.00 \mathrm{~A}$ $\mathrm{cm}^{-2}$. At $0.00 \mathrm{~A} \mathrm{~cm}^{-2}$, the AToF signal was reflected at the interface between the end-plate and flow channels/ribs. Due to the mismatch in physical properties, and hence acoustic impedance, the transition from end-plate to water in the flow channels produced a strong reflection peak. As current density increased, the flow channels were not only filled with water, but also with an increasing amount of oxygen bubbles. As outlined in Section 2.3, gas bubbles attenuate the AToF signal via resonant scattering. This explains why the averaged intensity decreased continuously with increasing current density. As more gas was produced, the acoustic input wave was scattered (anisotropically) more strongly, which reduced the intensity of the echo signal detected.

This also suggests that resonant scattering was the dominant attenuation mechanism in the flow channels. As current density increases, the amount of oxygen increases, and a number of flow regimes is typically observed in the flow channels. It is well-established that at low current densities the bubbly flow regime is observed, which then consecutively develops into slug and annular flow with increasing current density and location along any given flow channel [53]. Due to the large number of individual bubbles [31], the bubbly regime exhibits by far the highest amount of liquid/gas and gas/solid interfaces. If acoustic reflection at interfaces were to be the dominant attenuation mechanism, a maximum of reflection would be expected in the bubbly regime, with a decrease in reflection intensity for higher current densities due to the reduction in interfaces and the increasing amount of resonant scattering. However, as a continuous decrease in reflection intensity was observed experimentally, it 
appears likely that the dominant mechanism of acoustic attenuation in the flow channels was resonant scattering.

To establish the link between oxygen gas production and AToF signal, the pulse-echo response averaged across all channels (Figure 5 (b)) and the inverse (increasing gas flow reduces the reflection intensity) of the gravimetric gas flow per flow channel, $G$, (Figure 5 (c)) are displayed as a function of current density. The gas flow was calculated theoretically based on Faraday's law $[31,53]$. The different gas flows at the sensor locations was accounted for by adapting the active area used for the calculation, according to the location of the sensor along the flow channels. The pulse-echo response showed a continuous decrease with current density, which mirrors the behaviour of the inverse oxygen flow $1 / G$. An initial drop at low current densities was followed by a linear decrease from around $0.50 \mathrm{~A} \mathrm{~cm}^{-2}$ to $2.00 \mathrm{~A} \mathrm{~cm}^{-2}$. The distance between the curves for different locations was not as pronounced for the pulse-echo response as for the calculated inverse gas flow. This implies that attenuation due to resonant scattering was the dominant mechanism. However, it was convoluted by other attenuation mechanisms, such as reflection from gas/liquid and liquid/solid interfaces.

This was further supported by comparing the average reflection intensity in the flow channels under operation and in the completely dry cell. The band of average reflection intensity (two standard deviations) ranged between $11.46 \%$ and $20.75 \%$ (dashed black lines in Figure 5 (b)) for AToF measurements of the cell completely filled with air and void of water. However, the dry reflection intensity was considerably higher than values obtained during PEMWE operation at current densities beyond $0.75 \mathrm{~A} \mathrm{~cm}^{-2}$, where the flow channels contained a water-gas mixture. The reflection intensity obtained is influenced by two main factors: signal attenuation at interfaces and resonant scattering from bubbles and bubble clouds surrounded by water. While the attenuation at a solid-gas interface is higher than at a solid-liquid interface (see Section 2.3), resonant scattering is an additional mechanism of signal attenuation which is dependent on the gas fraction in the flow channels. Hence, in the dry cell there is very strong signal attenuation at the interface between end-plate and flow 
channels as the channels are only filled with air, but no resonant scattering as this requires gas bubbles in a liquid medium. Under operation of the PEMWE, on the other hand, the attenuation between endplate and flow channels is much less pronounced, as it is a solid-liquid interface. However, with increasing current density (gas fraction) the attenuation via resonant scattering increases, so that the overall measured reflection intensity decreases with current density and reaches values lower than for the dry cell. Hence, the signal attenuation in the flow channels could not be solely caused by the reflection at the gas interface. It is therefore implied that resonant scattering caused the unexpectedly low reflection intensity values.

Another feature of the AToF signal was that it exhibited an approximately parabolic profile across the flow channels. From left to right, the transition from a given acoustic intensity level to a lower one (e.g. blue to yellow in Figure 5 (a)) occurred at an increasingly high current density. Therefore, the lines of equal AToF intensity (black dashed lines in Figure 5 (a)) formed a parabolic profile roughly increasing from left to right. The effect of non-uniform flow distribution, following a parabolic profile, in a parallel flow-field is well documented [59-61] and has been observed with optical imaging of the PEMWE flow channels [19]. This is a consequence of the flow-field being designed in a Z-pattern, with water inlet and outlet being located at diagonally opposed ends of the parallel flow-field, which causes the water flow rate to be higher in the channels to the right (closer to the outlet) by a factor of up to five compared to the remainder of the channels [59]. This further established the ability of AToF to represent the physical processes in the flow-field. 


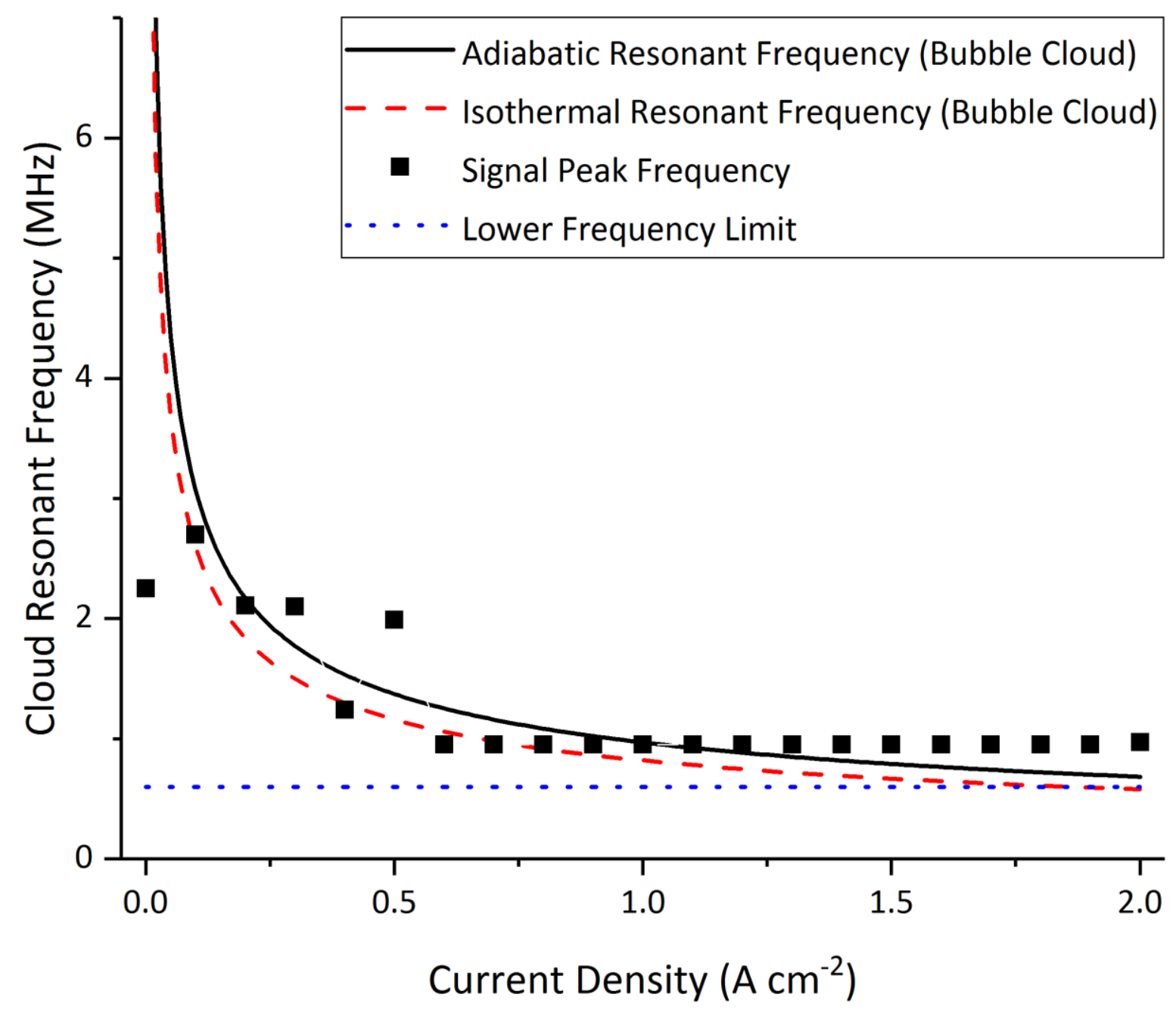

Fig. 6: The resonant scattering frequency of a bubble cloud with the diameter of a flow channel for isothermal and adiabatic operation of the PEMWE as a function of current density. The peak frequency of the AToF signal (obtained via FFT analysis) is shown as single data points, which are consistent with the resonant scattering frequency. The lower frequency detection limit is given for reference (dashed blue line). All calculations and measurements refer to the middle sensor location.

To further confirm resonant scattering as a dominant force of acoustic attenuation in the flow channels, for the middle location, the calculated frequencies for resonant scattering were compared with the peak of the signal frequency spectrum obtained via FFT (Figure 6). The frequency of resonant scattering was calculated as a function of current density using Equation (4) from Section 2.3, using a mass balance and Faraday's law to determine the gas fraction [31,53]. For the middle location, the active area was assumed to be half of the entire active area, thus only accounting for the amount of 
gas which had accumulated in the flow channels at the middle sensor location. Assuming the bubble cloud diameter being equal to the width of a flow channel, the resonant frequency is trending towards infinite values for $0.00 \mathrm{~A} \mathrm{~cm}^{-2}$ and falls to around $0.50 \mathrm{MHz}$ at $2.00 \mathrm{~A} \mathrm{~cm}^{-2}$. Additionally, the frequency of peak intensity was determined from the AToF signal for each current density via FFT analysis of the waveform along the entire observed time-of-flight. This indicated the most common echo-signal frequency occurring in the PEMWE and was linked to the processes causing the echo-response. The peaks related to the flow channels were by far the most dominant signal; hence, it is assumed that the obtained frequency spectrum was representative of the flow channel processes. Peak frequencies were observed in the range from around $2.70 \mathrm{MHz}$ to $0.95 \mathrm{MHz}$. The development of the observed peak frequency broadly followed the trend of the calculated bubble cloud frequency. This indicated that a major part of the received pulse-echo signal originated from the resonant scattering of bubbles and bubble clouds, respectively. Due to its anisotropy, this mechanism caused strong attenuation of the overall signal; however, the fraction of signal scattered towards the AToF sensor was still significant, as indicated by the measured and calculated frequency spectra.

\section{LGDL}

By increasing the acoustic gain and investigating a different time-of-flight interval, a detailed AToF investigation of the LGDL was made possible. The LGDL lacked clear macroscopic structures compared to the flow field (channels, ribs), but consisted of a high number of water or gas-filled pores and titanium particles. As for the flow-field, the LGDL was probed between $0.00 \mathrm{~A} \mathrm{~cm}^{-2}$ and $2.00 \mathrm{~A} \mathrm{~cm}^{-2}$ at upper, middle, and lower sensor locations. The resulting current (time) / location map is shown for the middle position in Figure 7 (a). The $x$-axis shows the line scan across the middle section and the $y$ axis the varying average cell current density as it increases with time. 

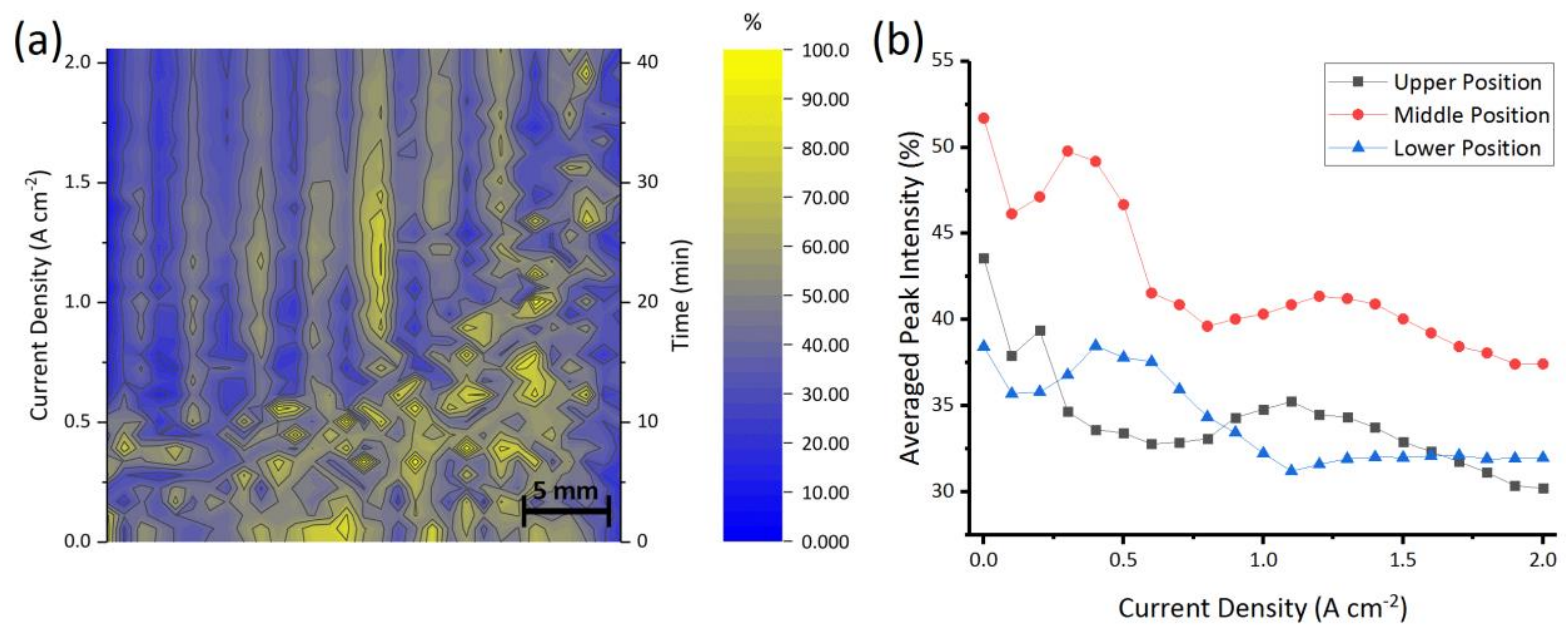

Fig. 7: (a) AToF reflection map across the LGDL width at the middle sensor location for current densities ranging from 0.00 $A \mathrm{~cm}^{-2}$ to $2.00 \mathrm{~A} \mathrm{~cm}^{-2}$. The intensity of the reflection peaks is indicated using a linear color map. (b) Average intensity of reflection peaks across the LGDL width for lower, middle, and upper sensor locations as a function of current density.

For low current densities below $0.50 \mathrm{~A} \mathrm{~cm}^{-2}$, randomly distributed reflection maxima and minima were observed, which is similar to Figure 4 (c). Above $0.50 \mathrm{~A} \mathrm{~cm}^{-2}$, this random distribution disappeared and a structure of clearly localized maxima and minima developed, which mirrored the rib/channel structure of the flow-field. It is well established through neutron imaging studies that the LGDL carries more oxygen gas and less water under the rib areas than under the channel areas of the flow-field in an operating PEMWE $[13,14,16]$. This trend was confirmed by AToF imaging, providing further confidence in this technique and establishing the possibility to examine the water-gas distribution in the LGDL in more detail via AToF. However, it has to be noted that the measurement of LGDL processes through AToF is affected by the flow-field. As the acoustic signal has to travel through the flow-field before reaching the LGDL, and the signal reflected by the LGDL has again to cross through the flowfield before being detected, processes in the flow-field can potentially lead to deviations in the signal associated with the LGDL, e.g. the existence of rib/channel features in the AToF response from the LGDL can potentially be an artefact caused by the input signal being transmitted through the flowfield before penetrating the LGDL. 
The overall intensity of reflection peaks attributed to the LGDL decreased with increasing current density (Figure 7 (b)). The variance between individual locations is likely caused by differences in local porosity of the LGDL and the formation of gas and water pathways in specific areas. As the local distribution of LGDL pores can vary across the active area, and it is well established that often preferential gas pathways are formed through the LGDL [12,62-64], these local non-uniformities in water and gas content in the LGDL might cause variations in the reflection intensity as observed in Figure 7 (b). However, this will need to be confirmed by further research.

A decrease from around $52 \%$ acoustic intensity at $0.00 \mathrm{~A} \mathrm{~cm}^{-2}$ to around $38 \%$ at $2.00 \mathrm{~A} \mathrm{~cm}^{-2}$ was observed for the middle position. However, the typical decrease in reflection peaks with current density was considerably smaller in the LGDL than in the flow channels. Other than in the flow-field, where almost the entire channel was filled with gas at high current density, in the LGDL only certain parts of the porosity of the LGDL contained gas at any given time. The remainder consisted of solid titanium and water pathways, which change little with current density.

The development of reflection intensity with current density observed here is consistent with throughplane water thickness measurements performed using neutron imaging [12]. Neutron imaging can reliably measure the absolute water thickness in a sample due to the strong interactions of neutrons with hydrogen atoms and therefore data on the water thickness in the LGDL obtained via neutron imaging can be used for validation and calibration of AToF data. Maier et al. [12] found a decrease in water thickness by $12 \%$ between $0.25 \mathrm{~A} \mathrm{~cm}^{-2}$ and $1.50 \mathrm{~A} \mathrm{~cm}^{-2}$ averaged across all rib areas using the same LGDL material as this work. This compares to a decrease of $13 \%$ in reflection intensity across the active area found in this work between $0.20 \mathrm{~A} \mathrm{~cm}^{-2}$ and $1.50 \mathrm{~A} \mathrm{~cm}^{-2}$. The close correlation in neutron imaging data and ATOF response emphasized the possibility of applying AToF imaging to LGDL processes and validated the chosen methodology. 


\section{Conclusion}

This works establishes AToF imaging as a powerful diagnostic tool for PEMWEs. It is demonstrated that the internal structure and assembly of flow-field and LGDL can be visualized using AToF scans across the PEMWE active area. This can be used for rapid, non-destructive inspection and diagnosis of PEMWE systems and offers a much lower cost and easily deployable operando imaging capability than established methods, such as X-ray or neutron imaging. By measuring the pulse-echo response of a PEMWE operated between $0.00 \mathrm{~A} \mathrm{~cm}^{-2}$ and $2.00 \mathrm{~A} \mathrm{~cm}^{-2}$ a range of effects typical for PEMWEs have been observed, which corroborates the effectiveness of AToF imaging. These effects were related to the flow-field as well as the LGDL.

Acoustic investigation of the flow-field showed a general decrease in reflection intensity with increasing current density. This indicates an increasing acoustic attenuation, which is caused by the increasing amount of oxygen gas produced and removed through the flow channels. It is shown by calculating the amount of oxygen produced due to electrochemical activity that the AToF signal scales closely with the inverse of the amount of gas in the flow channels. By determining the peak frequency of the AToF signal as a function of current density using FFT analysis, and comparing these data with theoretical expectations for the frequency of resonant scattering of bubbles, the link between gas bubbles in the flow channels and acoustic attenuation was confirmed. Further, AToF imaging yielded a parabolic profile of intensity across the flow channels, an effect well established in literature, which corroborates the validity of the data obtained in this work.

AToF imaging of the LGDL showed a granular distribution of reflection maxima and minima. However, the spatial resolution of the AToF equipment was not sufficient to resolve the LGDL microstructure of gas/water pathways and titanium particles. Just as for the flow-field, a general trend of decreasing reflection intensity with increasing current density was observed between $0.00 \mathrm{~A} \mathrm{~cm}^{-2}$ and $2.00 \mathrm{~A} \mathrm{~cm}^{-2}$. However, the trend was less pronounced than for the flow-field. It was demonstrated that the AToF response scales closely to data on the water thickness in the identical LGDL material, obtained via 
neutron imaging. Further, ATOF imaging showed a distinct difference between areas of the LGDL under rib and land areas of the flow-field, respectively. This can potentially be attributed to the accumulation of gas and water under rib and channels of the flow-field, respectively. This effect has been well documented by neutron imaging. Overall, this confirms the ability of AToF imaging to investigate macroscopic processes in the LGDL.

In conclusion, it was demonstrated that AToF imaging is well suited to investigate mass transport processes in flow-field as well as the LGDL of PEMWEs. It offers a low-cost, rapid, non-destructive, operando imaging tool. In a research context, it can potentially replace more time-consuming, expensive and specialized investigation techniques such as neutron and X-ray imaging. Commercial applications could include rapid inspection of single cells and PEMWE stacks or the diagnosis of nonuniform distribution of reactants across the active area, which could indicate faulty cell assembly or issues related to the CCM. Future work will focus on developing AToF techniques to selectively obtain data on the state of the CCM and develop lifetime predictions based on AToF data.

\section{Acknowledgements}

The authors acknowledge financial support into the EIL's hydrogen and fuel cell activity from the EPSRC through grants (EP/R023581/1; EP/P009050/1; EP/N032888/1; EP/M014371/1; EP/M009394; EP/L015749/1; EP/K038656/1). Support from the National Measurement System of the UK's Department of Business, Energy \& Industrial Strategy is also gratefully acknowledged. PRS acknowledges funding from The Royal Academy of Engineering (CiET1718/59).

\section{References}

[1] I. Staffell, D. Scamman, A. Velazquez Abad, P. Balcombe, P.E. Dodds, P. Ekins, N. Shah, K.R. Ward, The role of hydrogen and fuel cells in the global energy system, Energy Environ. Sci. 12 (2019) 463-491. doi:10.1039/c8ee01157e.

[2] M. Carmo, D.L. Fritz, J. Mergel, D. Stolten, A comprehensive review on PEM water electrolysis, Int. J. Hydrogen Energy. 38 (2013) 4901-4934. doi:10.1016/j.ijhydene.2013.01.151.

[3] S.M. Saba, M. Müller, M. Robinius, D. Stolten, The investment costs of electrolysis - A comparison of cost studies from the past 30 years, Int. J. Hydrogen Energy. 43 (2018) 1209- 
1223. doi:10.1016/j.ijhydene.2017.11.115.

[4] O. Schmidt, A. Gambhir, I. Staffell, A. Hawkes, J. Nelson, S. Few, Future cost and performance of water electrolysis: An expert elicitation study, Int. J. Hydrogen Energy. 42 (2017) 3047030492. doi:10.1016/j.ijhydene.2017.10.045.

[5] G. Yang, S. Yu, Z. Kang, Y. Li, G. Bender, B.S. Pivovar, J.B. Green, D.A. Cullen, F.Y. Zhang, Building Electron/Proton Nanohighways for Full Utilization of Water Splitting Catalysts, Adv. Energy Mater. 1903871 (2020) 1-10. doi:10.1002/aenm.201903871.

[6] C. Klose, T. Saatkamp, A. Münchinger, L. Bohn, G. Titvinidze, M. Breitwieser, K. Kreuer, S. Vierrath, All-Hydrocarbon MEA for PEM Water Electrolysis Combining Low Hydrogen Crossover and High Efficiency, Adv. Energy Mater. 1903995 (2020) 1-9. doi:10.1002/aenm.201903995.

[7] A. Buttler, H. Spliethoff, Current status of water electrolysis for energy storage, grid balancing and sector coupling via power-to-gas and power-to-liquids: A review, Renew. Sustain. Energy Rev. 82 (2018) 2440-2454. doi:10.1016/j.rser.2017.09.003.

[8] J.O. Majasan, F. lacoviello, J.I.S. Cho, M. Maier, X. Lu, T.P. Neville, I. Dedigama, P.R. Shearing, D.J.L. Brett, Correlative study of microstructure and performance for porous transport layers in polymer electrolyte membrane water electrolysers by X-ray computed tomography and electrochemical characterization, Int. J. Hydrogen Energy. 44 (2019) 19519-19532. doi:10.1016/j.ijhydene.2019.05.222.

[9] J.O. Majasan, F. lacoviello, P.R. Shearing, D.J. Brett, Effect of Microstructure of Porous Transport Layer on Performance in Polymer Electrolyte Membrane Water Electrolyser, Energy Procedia. 151 (2018) 111-119. doi:10.1016/j.egypro.2018.09.035.

[10] M. Suermann, K. Takanohashi, A. Lamibrac, T.J. Schmidt, F.N. Büchi, Influence of Operating Conditions and Material Properties on the Mass Transport Losses of Polymer Electrolyte Water Electrolysis, J. Electrochem. Soc. 164 (2017) F973-F980. doi:10.1149/2.13517109jes.

[11] L. Zielke, A. Fallisch, N. Paust, R. Zengerle, S. Thiele, Tomography based screening of flow field / current collector combinations for PEM water electrolysis, RSC Adv. 4 (2014) 58888-58894. doi:10.1039/C4RA12402B.

[12] M. Maier, J. Dodwell, R. Ziesche, C. Tan, T. Heenan, J. Majasan, N. Kardjilov, H. Markötter, I. Manke, L. Castanheira, G. Hinds, P.R. Shearing, D.J.L. Brett, Mass transport in polymer electrolyte membrane water electrolyser liquid-gas diffusion layers: A combined neutron imaging and X-ray computed tomography study, J. Power Sources. 455 (2020). doi:10.1016/j.jpowsour.2020.227968.

[13] O. Panchenko, E. Borgardt, W. Zwaygardt, F.J. Hackemüller, M. Bram, N. Kardjilov, T. Arlt, I. Manke, M. Müller, D. Stolten, W. Lehnert, In-situ two-phase flow investigation of different porous transport layer for a polymer electrolyte membrane (PEM) electrolyzer with neutron spectroscopy, J. Power Sources. 390 (2018) 108-115. doi:10.1016/j.jpowsour.2018.04.044.

[14] J. Seweryn, J. Biesdorf, T.J. Schmidt, P. Boillat, Communication-Neutron Radiography of the Water/Gas Distribution in the Porous Layers of an Operating Electrolyser, J. Electrochem. Soc. 163 (2016) F3009-F3011. doi:10.1149/2.0641607jes.

[15] O.F. Selamet, U. Pasaogullari, D. Spernjak, D.S. Hussey, D.L. Jacobson, M.D. Mat, Two-phase flow in a proton exchange membrane electrolyzer visualized in situ by simultaneous neutron radiography and optical imaging, Int. J. Hydrogen Energy. 38 (2013) 5823-5835. doi:10.1016/j.ijhydene.2013.02.087. 
[16] M.A. Hoeh, T. Arlt, N. Kardjilov, I. Manke, J. Banhart, D.L. Fritz, J. Ehlert, W. Lüke, W. Lehnert, In-operando Neutron Radiography Studies of Polymer Electrolyte Membrane Water Electrolyzers, ECS Trans. 69 (2015) 1135-1140.

[17] I. Dedigama, P. Angeli, K. Ayers, J.B. Robinson, P.R. Shearing, D. Tsaoulidis, D.J.L. Brett, In situ diagnostic techniques for characterisation of polymer electrolyte membrane water electrolysers - Flow visualisation and electrochemical impedance spectroscopy, Int. J. Hydrogen Energy. 39 (2014) 4468-4482. doi:10.1016/j.ijhydene.2014.01.026.

[18] I. Dedigama, P. Angeli, N. Van Dijk, J. Millichamp, D. Tsaoulidis, P.R. Shearing, D.J.L. Brett, Current density mapping and optical flow visualisation of a polymer electrolyte membrane water electrolyser, J. Power Sources. 265 (2014) 97-103. doi:10.1016/j.jpowsour.2014.04.120.

[19] J.O. Majasan, J.I.S. Cho, I. Dedigama, D. Tsaoulidis, P. Shearing, D.J.L. Brett, Two-phase flow behaviour and performance of polymer electrolyte membrane electrolysers: Electrochemical and optical characterisation, Int. J. Hydrogen Energy. 43 (2018) 15659-15672. doi:10.1016/j.ijhydene.2018.07.003.

[20] J. Mo, Z. Kang, G. Yang, Y. Li, S.T. Retterer, D.A. Cullen, T.J. Toops, G. Bender, B.S. Pivovar, J.B. Green, F.Y. Zhang, In situ investigation on ultrafast oxygen evolution reactions of water splitting in proton exchange membrane electrolyzer cells, J. Mater. Chem. A. 5 (2017) 18469-18475. doi:10.1039/c7ta05681h.

[21] T.M. Roberts, M. Talebzadeh, Acoustic emission monitoring of fatigue crack propagation, J. Constr. Steel Res. 59 (2003) 695-712. doi:10.1016/S0143-974X(02)00064-0.

[22] A. Nair, C.S. Cai, Acoustic emission monitoring of bridges: Review and case studies, Eng. Struct. 32 (2010) 1704-1714. doi:10.1016/j.engstruct.2010.02.020.

[23] T. Ohzuku, H. Tomura, K. Sawai, Monitoring of Particle Fracture by Acoustic Emission during Charge and Discharge of Li/MnO2 Cells, J. Electrochem. Soc. 144 (1997) 3496-3500. doi:10.1149/1.1838039.

[24] K. Rhodes, N. Dudney, E. Lara-Curzio, C. Daniel, Understanding the Degradation of Silicon Electrodes for Lithium-Ion Batteries Using Acoustic Emission, J. Electrochem. Soc. 157 (2010) A1354-A1360. doi:10.1149/1.3489374.

[25] N. Kircheva, S. Genies, D. Brun-Buisson, P.X. Thivel, Study of Solid Electrolyte Interface Formation and Lithium Intercalation in Li-Ion Batteries by Acoustic Emission, J. Electrochem. Soc. 159 (2012) A18-A25. doi:Doi 10.1149/2.045201jes.

[26] B. Dev, M.E. Walter, Comparative study of the leak characteristics of two ceramic/glass composite seals for solid oxide fuel cells, Fuel Cells. 15 (2015) 115-130. doi:10.1002/fuce.201400095.

[27] B. Dev, M.E. Walter, G.B. Arkenberg, S.L. Swartz, Mechanical and thermal characterization of a ceramic/glass composite seal for solid oxide fuel cells, J. Power Sources. 245 (2014) 958-966. doi:10.1016/j.jpowsour.2013.07.054.

[28] B. Legros, P.X. Thivel, Y. Bultel, M. Boinet, R.P. Nogueira, Acoustic emission: Towards a realtime diagnosis technique for Proton exchange membrane fuel cell operation, J. Power Sources. 195 (2010) 8124-8133. doi:10.1016/j.jpowsour.2010.07.045.

[29] B. Legros, P.X. Thivel, F. Druart, Y. Bultel, R. Nogueira, Diagnosis and Modelling of ProtonExchange-Membrane Fuel Cell via and Acoustic-Emission Measurements, in: Electromotion, 
Lille, 2009: pp. 1-6. doi:10.1109/ELECTROMOTION.2009.5259133.

[30] V.S. Bethapudi, M. Maier, G. Hinds, P.R. Shearing, D.J.L. Brett, M.O. Coppens, Acoustic emission as a function of polarisation: Diagnosis of polymer electrolyte fuel cell hydration state, Electrochem. Commun. 109 (2019) 106582. doi:10.1016/j.elecom.2019.106582.

[31] M. Maier, Q. Meyer, J. Majasan, C. Tan, I. Dedigama, J. Robinson, J. Dodwell, Y. Wu, L. Castanheira, G. Hinds, P.R. Shearing, D.J.L. Brett, Operando flow regime diagnosis using acoustic emission in a polymer electrolyte membrane water electrolyser, J. Power Sources. 424 (2019) 138-149. doi:10.1016/j.jpowsour.2019.03.061.

[32] M. Maier, Q. Meyer, J. Majasan, R.E. Owen, J.B. Robinson, J. Dodwell, Y. Wu, L. Castanheira, G. Hinds, P.R. Shearing, D.J.L. Brett, Diagnosing Stagnant Gas Bubbles in a Polymer Electrolyte Membrane Water Electrolyser Using Acoustic Emission, Front. Energy Res. 8 (2020). doi:10.3389/fenrg.2020.582919.

[33] B. Sood, M. Osterman, M. Pecht, Health monitoring of lithium-ion batteries, Proc. - 10th Annu. IEEE Symp. Prod. Compliance Eng. ISPCE 2013. (2013) 3-8. doi:10.1109/ISPCE.2013.6664165.

[34] A.G. Hsieh, S. Bhadra, B.J. Hertzberg, P.J. Gjeltema, A. Goy, J.W. Fleischer, D.A. Steingart, Electrochemical-acoustic time of flight: in operando correlation of physical dynamics with battery charge and health, Energy Environ. Sci. 8 (2015) 1569-1577. doi:10.1039/C5EE00111K.

[35] S. Bhadra, A.G. Hsieh, M.J. Wang, B.J. Hertzberg, D.A. Steingart, Anode Characterization in ZincManganese Dioxide AA Alkaline Batteries Using Electrochemical-Acoustic Time-of-Flight Analysis, J. Electrochem. Soc. 163 (2016) A1050-A1056. doi:10.1149/2.1201606jes.

[36] W. Chang, R. Mohr, A. Kim, A. Raj, G. Davies, K. Denner, J.H. Park, D. Steingart, Measuring effective stiffness of Li-ion batteries: Via acoustic signal processing, J. Mater. Chem. A. 8 (2020) 16624-16635. doi:10.1039/d0ta05552b.

[37] G. Davies, K.W. Knehr, B. Van Tassell, T. Hodson, S. Biswas, A.G. Hsieh, D.A. Steingart, State of Charge and State of Health Estimation Using Electrochemical Acoustic Time of Flight Analysis, J. Electrochem. Soc. 164 (2017) A2746-A2755. doi:10.1149/2.1411712jes.

[38] L. Gold, T. Bach, W. Virsik, A. Schmitt, J. Müller, T.E.M. Staab, G. Sextl, Probing lithium-ion batteries' state-of-charge using ultrasonic transmission - Concept and laboratory testing, J. Power Sources. 343 (2017) 536-544. doi:10.1016/j.jpowsour.2017.01.090.

[39] S. Guorong, L. Yaoqian, L. Yan, G. Jie, H. Cunfu, Estimating state of charge of lithium-ion batteries using ultrasonic reflection and transmission coefficients by Legendre orthogonal polynomial method, 2019 14th IEEE Int. Conf. Electron. Meas. Instruments, ICEMI 2019. (2019) 1256-1261. doi:10.1109/ICEMI46757.2019.9101585.

[40] P. Ladpli, F. Kopsaftopoulos, F.K. Chang, Estimating state of charge and health of lithium-ion batteries with guided waves using built-in piezoelectric sensors/actuators, J. Power Sources. 384 (2018) 342-354. doi:10.1016/j.jpowsour.2018.02.056.

[41] P. Ladpli, F. Kopsaftopoulos, R. Nardari, F.-K. Chang, Battery charge and health state monitoring via ultrasonic guided-wave-based methods using built-in piezoelectric transducers, Smart Mater. Nondestruct. Eval. Energy Syst. 2017. 10171 (2017) 1017108. doi:10.1117/12.2260107.

[42] J.B. Robinson, M. Maier, G. Alster, T. Compton, D.J.L. Brett, P.R. Shearing, Spatially resolved ultrasound diagnostics of Li-ion battery electrodes, Phys. Chem. Chem. Phys. (2019). doi:10.1039/C8CP07098A. 
[43] J.B. Robinson, M. Pham, M.D.R. Kok, T.M.M. Heenan, D.J.L. Brett, P.R. Shearing, Examining the Cycling Behaviour of Li-Ion Batteries Using Ultrasonic Time-of-Flight Measurements, J. Power Sources. 444 (2019) 227318. doi:10.1016/j.jpowsour.2019.227318.

[44] C. Bommier, Y. Lu, M. Williams, C. Bommier, W. Chang, Y. Lu, J. Yeung, G. Davies, In Operando Acoustic Detection of Lithium Metal Plating in Commercial LiCoO 2 / Graphite Pouch Cells In Operando Acoustic Detection of Lithium Metal Plating in Commercial LiCoO 2 / Graphite Pouch Cells, Cell Reports Phys. Sci. (n.d.) 100035. doi:10.1016/j.xcrp.2020.100035.

[45] J.B. Robinson, R.E. Owen, M. Kok, M. Maier, J. Majasan, M. Braglia, Ri. Stocker, T. Amietszajew, A. Roberts, R. Bhagat, D. Billson, J. Olson, J. Park, G. Hinds, A. Ahlberg Tiblad, P.R. Shearing, D. Brett, Identifying Defects in Li-lon Cells Using Ultrasound Acoustic Measurements, J. Electrochem. Soc. (2020). doi:10.1149/1945-7111/abb174.

[46] A.S. Aricò, S. Siracusano, N. Briguglio, V. Baglio, N. Van Dijk, H. Yildirim, D. Greenhalgh, L. Merlo, S. Tonella, L. Grahl-Madsen, G. Kielmann, S. Steinigeweg, EUROPEAN COMMISSION HORIZON 2020 PROGRAMME FUEL CELLS AND HYDROGEN JOINT UNDERTAKING ( FCH 2 JU ), 2016. http://hpem2gas.eu/download/public_reports/public_deliverables/HPEM2GAS-D2-1Protocols.pdf.

[47] D.J. McClements, Ultrasonic Characterisation of Emulsions and Suspensions, Adv. Colloid Interface Sci. 37 (1991) 33-72.

[48] P.A. Hwang, W.J. Teague, Low-frequency resonant scattering of bubble clouds, J. Atmos. Ocean. Technol. $17 \quad$ (2000) 847-853. doi:10.1175/15200426(2000)017<0847:LFRSOB>2.0.CO;2.

[49] A.B. Pandit, J. Varley, R.B. Thorpe, J.F. Davidson, Measurement of bubble size distribution: an acoustic technique, Chem. Eng. Sci. 47 (1992) 1079-1089. doi:10.1016/0009-2509(92)802333.

[50] A. Prosperetti, Bubble-related ambient noise in the ocean, J. Acoust. Soc. Am. 84 (1988) 10421054. doi:10.1121/1.396740.

[51] R.A. Roy, W. Carey, M. Nicholas, J. Schindall, L.A. Crum, Low-frequency scattering from submerged bubble clouds, J. Acoust. Soc. Am. 92 (1992) 2993-2996. doi:10.1121/1.404368.

[52] W.M. Carey, J.W. Fitzgerald, Low Frequency Noise from Breaking Waves, in: Kerman B.R. Nat. Phys. Sources Underw. Sound, Springer, Dordrecht, 1993.

[53] H. Ito, T. Maeda, A. Nakano, Y. Hasegawa, N. Yokoi, C.M. Hwang, M. Ishida, A. Kato, T. Yoshida, Effect of flow regime of circulating water on a proton exchange membrane electrolyzer, Int. J. Hydrogen Energy. 35 (2010) 9550-9560. doi:10.1016/j.ijhydene.2010.06.103.

[54] S.M. Steen, J. Mo, Z. Kang, G. Yang, F.Y. Zhang, Investigation of titanium liquid/gas diffusion layers in proton exchange membrane electrolyzer cells, Int. J. Green Energy. 14 (2017) 162170. doi:10.1080/15435075.2016.1253582.

[55] G. Chisholm, P.J. Kitson, N.D. Kirkaldy, L.G. Bloor, L. Cronin, 3D printed flow plates for the electrolysis of water: an economic and adaptable approach to device manufacture, Energy Environ. Sci. 7 (2014) 3026-3032. doi:10.1039/C4EE01426J.

[56] T. Schuler, T.J. Schmidt, F.N. Büchi, Polymer Electrolyte Water Electrolysis: Correlating Performance and Porous Transport Layer Structure: Part II. Electrochemical Performance Analysis, J. Electrochem. Soc. 166 (2019) F555-F565. doi:10.1149/2.1241908jes. 
[57] Z. Kang, J. Mo, G. Yang, S.T. Retterer, D.A. Cullen, T.J. Toops, J.B. Green Jr, M.M. Mench, F.-Y. Zhang, Investigation of thin/well-tunable liquid/gas diffusion layers exhibiting superior multifunctional performance in low-temperature electrolytic water splitting, Energy Environ. Sci. 10 (2017) 166-175. doi:10.1039/C6EE02368A.

[58] T.E. Springer, Characterization of Polymer Electrolyte Fuel Cells Using AC Impedance Spectroscopy, J. Electrochem. Soc. 143 (1996) 587. doi:10.1149/1.1836485.

[59] J. Nie, Y. Chen, S. Cohen, B.D. Carter, R.F. Boehm, Numerical and experimental study of threedimensional fluid flow in the bipolar plate of a PEM electrolysis cell, Int. J. Therm. Sci. 48 (2009) 1914-1922. doi:10.1016/j.ijthermalsci.2009.02.017.

[60] J. Wang, H. Wang, Discrete approach for flow field designs of parallel channel configurations in fuel cells, Int. J. Hydrogen Energy. 37 (2012) 10881-10897. doi:10.1016/j.ijhydene.2012.04.034.

[61] J. Wang, H. Wang, Flow-field designs of bipolar plates in PEM fuel cells: Theory and applications, Fuel Cells. 12 (2012) 989-1003. doi:10.1002/fuce.201200074.

[62] M.A. Hoeh, T. Arlt, I. Manke, J. Banhart, D.L. Fritz, W. Maier, W. Lehnert, In operando synchrotron $\mathrm{X}$-ray radiography studies of polymer electrolyte membrane water electrolyzers, Electrochem. Commun. 55 (2015) 55-59. doi:10.1016/j.elecom.2015.03.009.

[63] C.H. Lee, J. Hinebaugh, R. Banerjee, S. Chevalier, R. Abouatallah, R. Wang, A. Bazylak, Influence of limiting throat and flow regime on oxygen bubble saturation of polymer electrolyte membrane electrolyzer porous transport layers, Int. J. Hydrogen Energy. 42 (2017) 2724-2735. doi:10.1016/j.ijhydene.2016.09.114.

[64] F. Arbabi, A. Kalantarian, R. Abouatallah, R. Wang, J.S. Wallace, A. Bazylak, Feasibility study of using microfluidic platforms for visualizing bubble flows in electrolyzer gas diffusion layers, J. Power Sources. 258 (2014) 142-149. doi:10.1016/j.jpowsour.2014.02.042. 